\title{
Asymptotic Behaviors of a Delayed Nonautonomous Predator-Prey System Governed by Difference Equations
}

\author{
Lili Liu' ${ }^{1,2}$ and Zhijun Liu' ${ }^{1,2}$ \\ ${ }^{1}$ Key Laboratory of Biological Resources Protection and Utilization of Hubei Province, Hubei University for \\ Nationalities Enshi, Hubei 445000, China \\ ${ }^{2}$ Department of Mathematics, Hubei University for Nationalities Enshi, Hubei 445000, China
}

Correspondence should be addressed to Zhijun Liu, zhijun_liu47@hotmail.com

Received 3 May 2011; Accepted 18 September 2011

Academic Editor: Zhen Jin

Copyright (C) $2011 \mathrm{~L}$. Liu and Z. Liu. This is an open access article distributed under the Creative Commons Attribution License, which permits unrestricted use, distribution, and reproduction in any medium, provided the original work is properly cited.

Based on a predator-prey differential system with continuously distributed delays, we derive a corresponding difference version by using the method of a discretization technique. A good understanding of permanence of system and global attractivity of positive solutions of system is gained. An example and its numerical simulations are presented to substantiate our theoretical results.

\section{Introduction}

It is well known that the classical Lotka-Volterra differential equations for predator-prey interaction can be written as

$$
\begin{aligned}
& x^{\prime}(t)=x(t)[a-b x(t)-c y(t)], \\
& y^{\prime}(t)=y(t)[-d+p x(t)-q y(t)],
\end{aligned}
$$

where $x(0)>0, y(0)>0, x(t)$, and $y(t)$ are the population densities of prey and predator, respectively, at time $t$, and the coefficients $a, b, c, d, p, q$ are positive constants. $a$ is the intrinsic growth rate of prey species, and $d$ represents the death rate of predator species; $b, q$ and $c, p$, denote their self-inhibition and interaction rates, respectively. To account for time lag effects 
in their interaction, Cushing [1] suggested visualizing system (1.1) as a special case of the following more general system

$$
\begin{aligned}
& x^{\prime}(t)=x(t)\left[a-\int_{0}^{+\infty} x(t-\theta) d l_{1}(\theta)-\int_{0}^{+\infty} y(t-\theta) d l_{2}(\theta)\right], \\
& y^{\prime}(t)=y(t)\left[-d+\int_{0}^{+\infty} x(t-\theta) d l_{3}(\theta)-\int_{0}^{+\infty} y(t-\theta) d l_{4}(\theta)\right],
\end{aligned}
$$

where $l_{i}(\theta)$ are nondecreasing functions on $[0,+\infty)$ with $\int_{0}^{+\infty} d l_{i}(\theta)<+\infty$ for each $i=1, \ldots, 4$, and with $\lim _{\theta \rightarrow 0^{+}}\left[l_{i}(\theta)-l_{i}(0)\right]$, respectively, equal to $b, c, p, q$ for $i=1, \ldots, 4$.

As Hale [2] pointed out, one expects that the lag effects tend to diminish gradually in ever-moderating pace as one moves backward in time after the initial instantaneous effect described by the assumption $\lim _{\theta \rightarrow 0^{+}}\left[l_{i}(\theta)-l_{i}(0)\right]>0$. The effects are negligible after a certain length of time $\tau>0$. Mathematically, for $\theta>0$, it is reasonable that $d l_{i}(\theta)$ can be assumed to be of the form $d l_{i}(\theta)=k_{i}(\theta) d \theta, i=1, \ldots, 4$, where

$\left(S_{1}\right) k_{i}(s) \geq 0$ and continuous for $s \in[0, \tau] ; k_{i}(s)=0$ for $s \in[\tau,+\infty]$;

$\left(S_{2}\right) k_{i}^{\prime}(s) \leq 0$ and continuous for $s \in(0, \tau) ; \lim _{s \rightarrow 0^{+}} k_{i}^{\prime}(s)$ and $\lim _{s \rightarrow \tau^{-}} k_{i}^{\prime}(s)$ both exist;

$\left(S_{3}\right) k_{i}^{\prime \prime}(s) \geq 0$ and continuous for $s \in(0, \tau)$.

The assumption $\left(S_{2}\right)$ represents gradual diminishing effect as one moves backward in time, and the assumption $\left(S_{3}\right)$ stands for the moderating pace of the decrease (see [2] or [3] for the assumptions $\left(S_{1}\right)$ to $\left(S_{3}\right)$ ). Leung [4] revised system (1.2) and established the following predator-prey model with continuously distributed delays

$$
\begin{aligned}
& x^{\prime}(t)=x(t)\left[a-b x(t)-c y(t)-\int_{0}^{\tau} k_{1}(s) x(t-s) d s-\int_{0}^{\tau} k_{2}(s) y(t-s) d s\right], \\
& y^{\prime}(t)=y(t)\left[-d+p x(t)-q y(t)+\int_{0}^{\tau} k_{3}(s) x(t-s) d s-\int_{0}^{\tau} k_{4}(s) y(t-s) d s\right] .
\end{aligned}
$$

The parameters of system (1.3) are assumed to be constant; however, in the real world the parameters are not fixed constants owing to the variation of environment. The effect of a varying environment is significant for evolutionary theory as the selective forces on systems in a fluctuating environment differ from those in a stable environment. Thus, it is realistic to assume that the parameters of system (1.3) are continuous functions with respect to $t$, and then we obtain the following form:

$$
\begin{aligned}
& x^{\prime}(t)=x(t)\left[a(t)-b(t) x(t)-c(t) y(t)-\int_{0}^{\tau} k_{1}(s) x(t-s) d s-\int_{0}^{\tau} k_{2}(s) y(t-s) d s\right], \\
& y^{\prime}(t)=y(t)\left[-d(t)+p(t) x(t)-q(t) y(t)+\int_{0}^{\tau} k_{3}(s) x(t-s) d s-\int_{0}^{\tau} k_{4}(s) y(t-s) d s\right],
\end{aligned}
$$

where the coefficients $a(t), b(t), c(t), d(t), p(t), q(t)$ are positive continuous functions and $k_{i}(s) d s$ satisfy $\int_{0}^{\tau} k_{i}(s) d s=1$ for $i=1, \ldots, 4$. 
Although much progress on the predator-prey models with discrete or distributed delays has been made, such models are not sufficiently researched in the sense that most results are continuous time versions related (see [5-13]). Many authors have argued that the discrete time models governed by difference equations are more appropriate than the continuous time ones when populations have a short life expectancy, nonoverlapping generations in the real world (see [14-18]). Meanwhile, discrete time models can provide efficient computational models of continuous time models for numerical simulations. To the best of our knowledge, no such work has been done for the corresponding discrete version of system (1.4).

In the following, we employ the discretization technique to derive the discrete version of system (1.4). Throughout this paper, let $\mathbb{Z}, \mathbb{Z}^{+}$and $\mathbb{R}^{2}$ denote the sets of all integers, nonnegative integers, and two-dimensional vector space, respectively. We begin to approximate the continuous time system by replacing the integral term with discrete sums of the form

$$
\int_{0}^{\tau} k_{i}(s) x(t-s) d s \approx \sum_{[s / h]=0}^{M} k_{i}\left(\left[\frac{s}{h}\right] h\right) x\left(\left[\frac{t}{h}\right] h-\left[\frac{s}{h}\right] h\right) w(h)
$$

for $t \in[n h,(n+1) h), s \in[r h,(r+1) h), n, r \in \mathbb{Z}^{+}, i=1, \ldots, 4$, where [-] denotes the greatest integer function. $M=[\tau / h], w(h)=h+O(h)$ for $h>0$, where the fixed number $h$ denotes an uniform discretization step size. We approximate system (1.4) by differential equations with piecewise constant arguments of the form

$$
\begin{aligned}
x^{\prime}(t)=x(t)[ & a\left(\left[\frac{t}{h}\right] h\right)-b\left(\left[\frac{t}{h}\right] h\right) x\left(\left[\frac{t}{h}\right] h\right)-c\left(\left[\frac{t}{h}\right] h\right) y\left(\left[\frac{t}{h}\right] h\right) \\
& \left.-\sum_{[s / h]=0}^{M} k_{1}\left(\left[\frac{s}{h}\right] h\right) x\left(\left[\frac{t}{h}\right] h-\left[\frac{s}{h}\right] h\right) h-\sum_{[s / h]=0}^{M} k_{2}\left(\left[\frac{s}{h}\right] h\right) y\left(\left[\frac{t}{h}\right] h-\left[\frac{s}{h}\right] h\right) h\right], \\
y^{\prime}(t)=y(t)[ & -d\left(\left[\frac{t}{h}\right] h\right)+p\left(\left[\frac{t}{h}\right] h\right) x\left(\left[\frac{t}{h}\right] h\right)-q\left(\left[\frac{t}{h}\right] h\right) y\left(\left[\frac{t}{h}\right] h\right) \\
& \left.+\sum_{[s / h]=0}^{M} k_{3}\left(\left[\frac{s}{h}\right] h\right) x\left(\left[\frac{t}{h}\right] h-\left[\frac{s}{h}\right] h\right) h-\sum_{[s / h]=0}^{M} k_{4}\left(\left[\frac{s}{h}\right] h\right) y\left(\left[\frac{t}{h}\right] h-\left[\frac{s}{h}\right] h\right) h\right]
\end{aligned}
$$

for $t \in[n h,(n+1) h), s \in[r h,(r+1) h), n, r \in \mathbb{Z}^{+}$. Noting that $[t / h]=n,[s / h]=r$, we integrate (1.6) over $[n h, t)$, where $t<(n+1) h$, then (1.6) can be reformulated as

$$
\begin{aligned}
& x^{\prime}(t)=x(t)\left[a(n h)-b(n h) x(n h)-c(n h) y(n h)-\sum_{r=0}^{M} k_{1}(r h) x(n h-r h) h-\sum_{r=0}^{M} k_{2}(r h) y(n h-r h) h\right] \\
& y^{\prime}(t)=y(t)\left[-d(n h)+p(n h) x(n h)-q(n h) y(n h)+\sum_{r=0}^{M} k_{3}(r h) x(n h-r h) h-\sum_{r=0}^{M} k_{4}(r h) y(n h-r h) h\right] .
\end{aligned}
$$


Denoting $x(n)=x(n h), y(n)=y(n h), a(n)=a(n h), b(n)=b(n h), c(n)=c(n h), d(n)=d(n h)$, $p(n)=p(n h), q(n)=q(n h), k_{i}(r)=k_{i}(r h) h, i=1, \ldots, 4$, then we have

$$
\begin{aligned}
& x(t)=x(n) \exp \left\{\left[a(n)-b(n) x(n)-c(n) y(n)-\sum_{r=0}^{M} k_{1}(r) x(n-r)-\sum_{r=0}^{M} k_{2}(r) y(n-r)\right](t-n h)\right\}, \\
& y(t)=y(n) \exp \left\{\left[-d(n)+p(n) x(n)-q(n) y(n)+\sum_{r=0}^{M} k_{3}(r) x(n-r)-\sum_{r=0}^{M} k_{4}(r) y(n-r)\right](t-n h)\right\} .
\end{aligned}
$$

Setting $t \rightarrow(n+1) h$ in (1.8) and simplifying, we get a discrete time analogue of continuous time system (1.4) with the form

$$
\begin{aligned}
& x(n+1)=x(n) \exp \left\{a(n)-b(n) x(n)-c(n) y(n)-\sum_{r=0}^{M} k_{1}(r) x(n-r)-\sum_{r=0}^{M} k_{2}(r) y(n-r)\right\} \\
& y(n+1)=y(n) \exp \left\{-d(n)+p(n) x(n)-q(n) y(n)+\sum_{r=0}^{M} k_{3}(r) x(n-r)-\sum_{r=0}^{M} k_{4}(r) y(n-r)\right\} .
\end{aligned}
$$

Our main purpose of this paper is to derive a set of easily verifiable sufficient conditions concerning the permanence and global attractivity of system (1.9). For convenience, we introduce the following definitions and notations.

Definition 1.1. It is said that system (1.9) is permanent if there exist positive constants $m_{i}, M_{i}$, $i=1,2$ and any positive solution $\left\{(x(n), y(n))^{\top}\right\}$ of system (1.9) satisfies

$$
\begin{aligned}
& m_{1} \leq \lim \inf _{n \rightarrow+\infty} x(n) \leq \lim \sup _{n \rightarrow+\infty} x(n) \leq M_{1}, \\
& m_{2} \leq \lim \inf _{n \rightarrow+\infty} y(n) \leq \lim \sup _{n \rightarrow+\infty} y(n) \leq M_{2} .
\end{aligned}
$$

Definition 1.2. A positive solution $\left\{(x(n), y(n))^{\top}\right\}$ of system (1.9) is global attractive if each other positive solution $\left\{\left(x^{*}(n), y^{*}(n)\right)^{\top}\right\}$ of system (1.9) satisfies

$$
\lim _{n \rightarrow+\infty}\left|x(n)-x^{*}(n)\right|=0, \quad \lim _{n \rightarrow+\infty}\left|y(n)-y^{*}(n)\right|=0 .
$$

Let $f(n)$ be a bounded sequence; we denote

$$
f^{u}=\sup _{n \in \mathbb{Z}^{+}}\{f(n)\}, \quad f^{l}=\inf _{n \in \mathbb{Z}^{+}}\{f(n)\} .
$$

Meanwhile, we make a convention that $\prod_{n=m}^{n=k} f(n)=1$ for all $m>k$.

This paper proceeds as follows. System (1.9) is analyzed to study the permanence and global attractivity of system (1.9) in the next two sections, respectively. In the final section, we 
give an example, and its numerical simulations are presented to substantiate our theoretical results.

\section{Permanence of System (1.9)}

In this section, we devote to investigating the permanence of system (1.9). To do so, we introduce the following lemmas.

Lemma 2.1 (see [19]). Assume that $\{x(n)\}$ satisfies $x(n)>0$ and

$$
x(n+1) \leq x(n) \exp [r(n)(1-a x(n))]
$$

for $n \in\left[n_{1},+\infty\right)$, where $a$ is a positive constant and $n_{1} \in \mathbb{Z}^{+}$. Then

$$
\lim _{n \rightarrow+\infty} \sup x(n) \leq \frac{1}{a r^{u}} \exp \left(r^{u}-1\right)
$$

Lemma 2.2 (see [19]). Assume that $\{x(n)\}$ satisfies $x(n)>0$ and

$$
x(n+1) \geq x(n) \exp [r(n)(1-\operatorname{ax}(n))]
$$

for $n \in\left[n_{2},+\infty\right)$, limsup $\sup _{n \rightarrow+\infty} x(n) \leq H$ and $x\left(n_{2}\right)>0$, where $a$ is a positive constant such that $a H>1$ and $n_{2} \in \mathbb{Z}^{+}$. Then

$$
\lim _{n \rightarrow+\infty} \inf x(n) \geq \frac{1}{a} \exp \left[r^{u}(1-a H)\right]
$$

For the simplicity of description, we denote

$$
\begin{gathered}
\Delta_{1}^{\varepsilon}=-d^{l}+\left[p^{u}+(M+1) k_{3}^{u}\right]\left(M_{1}+\varepsilon\right), \\
\Delta_{2}^{\varepsilon}=a^{l}-c^{u}\left(M_{2}+\varepsilon\right)-(M+1)\left[k_{1}^{u}\left(M_{1}+\varepsilon\right)+k_{2}^{u}\left(M_{2}+\varepsilon\right)\right], \\
\Delta_{3}^{\varepsilon}=-d^{u}+p^{l}\left(m_{1}-\varepsilon\right)+(M+1)\left[k_{3}^{l}\left(m_{1}-\varepsilon\right)-k_{4}^{u}\left(M_{2}+\varepsilon\right)\right], \\
\Delta_{i}=\lim _{\varepsilon \rightarrow 0} \Delta_{i}^{\varepsilon}, \quad i=1,2,3,
\end{gathered}
$$

where $M_{1}, M_{2}, m_{1}$ are, respectively, defined in (2.8) and (2.11)-(2.14) and $\varepsilon$ is a sufficient small positive constant. Now, we begin to search the conditions for the permanence of (1.9).

Theorem 2.3. If the conditions

$$
\min \left\{\Delta_{i}, i=1,2,3\right\}>0
$$

hold, then (1.9) is permanent. 
Proof. It is easy to verify that $\Omega=\left\{(x(n), y(n))^{\top} \mid x(n)>0, y(n)>0\right\}$ is a positive invariant set of (1.9) with $x(0)>0, y(0)>0$. Suppose that $\left\{(x(n), y(n))^{\top}\right\}$ is any positive solution of (1.9), we prove Theorem 2.3 by the following two steps.

Step 1 . We show that $\left\{(x(n), y(n))^{\top}\right\}$ is uniformly ultimately upper bounded.

From the first equation of (1.9), we have

$$
x(n+1) \leq x(n) \exp [a(n)-b(n) x(n)] \leq x(n) \exp \left\{a(n)\left[1-\frac{b^{l}}{a^{u}} x(n)\right]\right\} .
$$

Applying Lemma 2.1, we can obtain that

$$
\lim _{n \rightarrow+\infty} \sup x(n) \leq \frac{\exp \left(a^{u}-1\right)}{b^{l}} \stackrel{\text { def }}{=} M_{1} .
$$

From (2.8), there exists a sufficient large $n_{1} \in \mathbb{Z}^{+}$and any constant $\varepsilon>0$ such that

$$
x(n) \leq M_{1}+\varepsilon, \quad n \geq n_{1} .
$$

It follows from the second equation of (1.9) that

$$
\begin{aligned}
y(n+1) & \leq y(n) \exp \left\{-d^{l}+\left[p^{u}+(M+1) k_{3}^{u}\right]\left(M_{1}+\varepsilon\right)-q^{l} y(n)\right\} \\
& =y(n) \exp \left\{\Delta_{1}^{\varepsilon}\left[1-\frac{q^{l}}{\Delta_{1}^{\varepsilon}} y(n)\right]\right\} .
\end{aligned}
$$

So by Lemma 2.1 and letting $\varepsilon \rightarrow 0$ in (2.10), we have

$$
\lim _{n \rightarrow+\infty} \sup y(n) \leq \frac{\exp \left(\Delta_{1}-1\right)}{q^{l}} \stackrel{\text { def }}{=} M_{2} \text {. }
$$

Step 2. We prove that $\left\{(x(n), y(n))^{\top}\right\}$ is uniformly ultimately lower bounded.

For any sufficient small $\varepsilon>0$, it follows from (2.6) that $\Delta_{2}^{\varepsilon}>0$. According to (2.11), there exists an $n_{0}>n_{1}$ such that $y(n) \leq M_{2}+\varepsilon$ for $n \geq n_{0}$ and the above constant $\varepsilon$. By the first equation of (1.9), it gives that

$$
\begin{aligned}
x(n+1) & \geq x(n) \exp \left\{a^{l}-c^{u}\left(M_{2}+\varepsilon\right)-(M+1)\left[k_{1}^{u}\left(M_{1}+\varepsilon\right)+k_{2}^{u}\left(M_{2}+\varepsilon\right)\right]-b^{u} x(n)\right\} \\
& =x(n) \exp \left\{\Delta_{2}^{\varepsilon}\left[1-\frac{b^{u}}{\Delta_{2}^{\varepsilon}} x(n)\right]\right\} .
\end{aligned}
$$

Note that

$$
\frac{b^{u}}{\Delta_{2}^{\varepsilon}} M_{1}=\frac{b^{u}}{\Delta_{2}^{\varepsilon}} \frac{\exp \left(a^{u}-1\right)}{b^{l}} \geq \frac{b^{u}}{a^{l}} \frac{\exp \left(a^{u}-1\right)}{b^{l}}>\frac{\exp \left(a^{u}-1\right)}{a^{u}}>1,
$$


where we employ the inequality result that $\exp (x-1)>x$ for $x>0$. Therefore, applying Lemma 2.2 and setting $\varepsilon \rightarrow 0$, one has

$$
\lim _{n \rightarrow+\infty} \inf x(n) \geq \frac{\Delta_{2}}{b^{u}} \exp \left[\Delta_{2}\left(1-\frac{b^{u}}{\Delta_{2}} M_{1}\right)\right] \stackrel{\text { def }}{=} m_{1} .
$$

For the above constant $\varepsilon>0$, it follows from (2.14) that there exists a sufficient large $n_{1}^{*} \in \mathbb{Z}^{+}$ such that

$$
x(n) \geq m_{1}-\varepsilon, \quad n \geq n_{1}^{*} .
$$

From the second equation of (1.9), we have

$$
\begin{aligned}
y(n+1) & \geq y(n) \exp \left\{-d^{u}+p^{l}\left(m_{1}-\varepsilon\right)+(M+1)\left[k_{3}^{l}\left(m_{1}-\varepsilon\right)-k_{4}^{u}\left(M_{2}+\varepsilon\right)\right]-q^{u} y(n)\right\} \\
& =y(n) \exp \left\{\Delta_{3}^{\varepsilon}\left[1-\frac{q^{u}}{\Delta_{3}^{\varepsilon}} y(n)\right]\right\} .
\end{aligned}
$$

Clearly,

$$
\frac{q^{u}}{\Delta_{3}^{\varepsilon}} M_{2}=\frac{q^{u}}{\Delta_{3}^{\varepsilon}} \frac{\exp \left(\Delta_{1}-1\right)}{q^{l}} \geq \frac{q^{l} \exp \left\{-d^{u}+\left[p^{l}+(M+1) k_{3}^{l}\right] m_{1}-1\right\}}{\left[-d^{u}+\left(p^{l}+(M+1) k_{3}^{l}\right) m_{1}\right] q^{l}}>1 .
$$

Thus, applying Lemma 2.2 and letting $\varepsilon \rightarrow 0$, one has

$$
\lim _{n \rightarrow+\infty} \inf y(n) \geq \frac{\Delta_{3}}{q^{u}} \exp \left[\Delta_{3}\left(1-\frac{q^{u}}{\Delta_{3}} M_{2}\right)\right] \stackrel{\text { def }}{=} m_{2} .
$$

Based on the above Step 1 with Step 2, we can see that system (1.9) is permanent. This completes the proof of Theorem 2.3.

\section{Global Attractivity of Positive Solutions of System (1.9)}

In this section, we investigate the global attractivity of positive solutions of system (1.9).

Theorem 3.1. Assume that there exists a positive constant $\eta$ such that

$$
\begin{aligned}
& \min \left\{b^{l}, \frac{2}{M_{1}}-b^{u}\right\}-(M+1)\left(k_{1}^{u}+k_{3}^{u}\right)-p^{u} \geq \eta \\
& \min \left\{q^{l}, \frac{2}{M_{2}}-q^{u}\right\}-(M+1)\left(k_{2}^{u}+k_{4}^{u}\right)-c^{u} \geq \eta
\end{aligned}
$$

hold. Then any positive solution $\left\{(x(n), y(n))^{\top}\right\}$ of system (1.9) is global attractive. 
Proof. Denote $\left\{\left(x^{*}(n), y^{*}(n)\right)^{\top}\right\}$ be any other positive solution of system (1.9). Let $V_{11}(n)=$ $\left|\ln x(n)-\ln x^{*}(n)\right|$, then it follows from the first equation of (1.9) that

$$
\begin{aligned}
V_{11}(n+1)=\left|\ln x(n+1)-\ln x^{*}(n+1)\right| \\
\leq\left|\ln x(n)-\ln x^{*}(n)-b(n)\left(x(n)-x^{*}(n)\right)\right| \\
\quad+c(n)\left|y(n)-y^{*}(n)\right| \\
\quad+\sum_{r=0}^{M} k_{1}(r)\left|x(n-r)-x^{*}(n-r)\right| \\
\quad+\sum_{r=0}^{M} k_{2}(r)\left|y(n-r)-y^{*}(n-r)\right| .
\end{aligned}
$$

By the Mean Value theorem, we get

$$
\ln x(n)-\ln x^{*}(n)=\frac{1}{\theta_{1}(n)}\left(x(n)-x^{*}(n)\right),
$$

where $\theta_{1}(n)$ lies between $x(n)$ and $x^{*}(n)$. Then we have

$$
\begin{aligned}
\mid \ln & x(n)-\ln x^{*}(n)-b(n)\left(x(n)-x^{*}(n)\right) \mid \\
\quad & \left|\ln x(n)-\ln x^{*}(n)\right|-\left|\ln x(n)-\ln x^{*}(n)\right|+\left|\ln x(n)-\ln x^{*}(n)-b(n)\left(x(n)-x^{*}(n)\right)\right| \\
& =\left|\ln x(n)-\ln x^{*}(n)\right|-\frac{1}{\theta_{1}(n)}\left|x(n)-x^{*}(n)\right|+\left|\frac{1}{\theta_{1}(n)}\left(x(n)-x^{*}(n)\right)-b(n)\left(x(n)-x^{*}(n)\right)\right| \\
& =\left|\ln x(n)-\ln x^{*}(n)\right|-\left(\frac{1}{\theta_{1}(n)}-\left|\frac{1}{\theta_{1}(n)}-b(n)\right|\right)\left|x(n)-x^{*}(n)\right| .
\end{aligned}
$$

Combining (3.2) with (3.4), we have

$$
\begin{aligned}
\Delta V_{11}(n)= & V_{11}(n+1)-V_{11}(n) \\
\leq & -\left(\frac{1}{\theta_{1}(n)}-\left|\frac{1}{\theta_{1}(n)}-b(n)\right|\right)\left|x(n)-x^{*}(n)\right|+c(n)\left|y(n)-y^{*}(n)\right| \\
& +\sum_{r=0}^{M} k_{1}(r)\left|x(n-r)-x^{*}(n-r)\right|+\sum_{r=0}^{M} k_{2}(r)\left|y(n-r)-y^{*}(n-r)\right| .
\end{aligned}
$$

Next, we let

$$
V_{12}(n)=\sum_{r=0}^{M} \sum_{s=n-r}^{n-1} k_{1}(r)\left|x(s)-x^{*}(s)\right|+\sum_{r=0}^{M} \sum_{s=n-r}^{n-1} k_{2}(r)\left|y(s)-y^{*}(s)\right|
$$


Then we have

$$
\begin{aligned}
\Delta V_{12}(n)= & V_{12}(n+1)-V_{12}(n) \\
= & \sum_{r=0}^{M} \sum_{s=n+1-r}^{n} k_{1}(r)\left|x(s)-x^{*}(s)\right|-\sum_{r=0}^{M} \sum_{s=n-r}^{n-1} k_{1}(r)\left|x(s)-x^{*}(s)\right| \\
& +\sum_{r=0}^{M} \sum_{s=n+1-r}^{n} k_{2}(r)\left|y(s)-y^{*}(s)\right|-\sum_{r=0}^{M} \sum_{s=n-r}^{n-1} k_{2}(r)\left|y(s)-y^{*}(s)\right| \\
= & \sum_{r=0}^{M} \sum_{s=n+1-r}^{n-1} k_{1}(r)\left|x(s)-x^{*}(s)\right|+\sum_{r=0}^{M} k_{1}(r)\left|x(n)-x^{*}(n)\right| \\
& -\sum_{r=0}^{M} \sum_{s=n+1-r}^{n-1} k_{1}(r)\left|x(s)-x^{*}(s)\right|-\sum_{r=0}^{M} k_{1}(r)\left|x(n-r)-x^{*}(n-r)\right| \\
& +\sum_{r=0}^{M} \sum_{s=n+1-r}^{n-1} k_{2}(r)\left|y(s)-y^{*}(s)\right|+\sum_{r=0}^{M} k_{2}(r)\left|y(n)-y^{*}(n)\right| \\
& -\sum_{r=0}^{M} \sum_{s=n+1-r}^{n-1} k_{2}(r)\left|y(s)-y^{*}(s)\right|-\sum_{r=0}^{M} k_{2}(r)\left|y(n-r)-y^{*}(n-r)\right| \\
= & \sum_{r=0}^{M} k_{1}(r)\left|x(n)-x^{*}(n)\right|-\sum_{r=0}^{M} k_{1}(\mathrm{r})\left|x(n-r)-x^{*}(n-r)\right| \\
& +\sum_{r=0}^{M} k_{2}(r)\left|y(n)-y^{*}(n)\right|-\sum_{r=0}^{M} k_{2}(r)\left|y(n-r)-y^{*}(n-r)\right| .
\end{aligned}
$$

We set

$$
V_{1}(n)=V_{11}(n)+V_{12}(n),
$$

then it follows from (3.5) and (3.7) that

$$
\begin{aligned}
\Delta V_{1}(n)= & \Delta V_{11}(n)+\Delta V_{12}(n) \\
\leq & -\left(\frac{1}{\theta_{1}(n)}-\left|\frac{1}{\theta_{1}(n)}-b(n)\right|-\sum_{r=0}^{M} k_{1}(r)\right)\left|x(n)-x^{*}(n)\right| \\
& +\left(c(n)+\sum_{r=0}^{M} k_{2}(r)\right)\left|y(n)-y^{*}(n)\right|
\end{aligned}
$$

Similarly, we define

$$
V_{2}(n)=V_{21}(n)+V_{22}(n),
$$


where

$$
\begin{aligned}
V_{21}(n) & =\sum_{r=0}^{M} \sum_{s=n-r}^{n-1} k_{3}(r)\left|x(s)-x^{*}(s)\right| \\
& +\sum_{r=0}^{M} \sum_{s=n-r}^{n-1} k_{4}(r)\left|y(s)-y^{*}(s)\right|, \\
V_{22}(n) & =\left|\ln y(n)-\ln y^{*}(n)\right| .
\end{aligned}
$$

Then we have

$$
\begin{aligned}
\Delta V_{21}(n)= & V_{21}(n+1)-V_{21}(n) \\
= & \sum_{r=0}^{M} \sum_{s=n+1-r}^{n} k_{3}(r)\left|x(s)-x^{*}(s)\right|-\sum_{r=0}^{M} \sum_{s=n-r}^{n-1} k_{3}(r)\left|x(s)-x^{*}(s)\right| \\
& +\sum_{r=0}^{M} \sum_{s=n+1-r}^{n} k_{4}(r)\left|y(s)-y^{*}(s)\right|-\sum_{r=0}^{M} \sum_{s=n-r}^{n-1} k_{4}(r)\left|y(s)-y^{*}(s)\right| \\
= & \sum_{r=0}^{M} \sum_{s=n+1-r}^{n-1} k_{3}(r)\left|x(s)-x^{*}(s)\right|+\sum_{r=0}^{M} k_{3}(r)\left|x(n)-x^{*}(n)\right| \\
& -\sum_{r=0}^{M} \sum_{s=n+1-r}^{n-1} k_{3}(r)\left|x(s)-x^{*}(s)\right|-\sum_{r=0}^{M} k_{3}(r)\left|x(n-r)-x^{*}(n-r)\right| \\
& +\sum_{r=0}^{M} \sum_{s=n+1-r}^{n-1} k_{4}(r)\left|y(s)-y^{*}(s)\right|+\sum_{r=0}^{M} k_{4}(r)\left|y(n)-y^{*}(n)\right| \\
& -\sum_{r=0}^{M} \sum_{s=n+1-r}^{n-1} k_{4}(r)\left|y(s)-y^{*}(s)\right|-\sum_{r=0}^{M} k_{4}(r)\left|y(n-r)-y^{*}(n-r)\right| \\
= & \sum_{r=0}^{M} k_{3}(r)\left|x(n)-x^{*}(n)\right|-\sum_{r=0}^{M} k_{3}(r)\left|x(n-r)-x^{*}(n-r)\right| \\
& +\sum_{r=0}^{M} k_{4}(r)\left|y(n)-y^{*}(n)\right|-\sum_{r=0}^{M} k_{4}(r)\left|y(n-r)-y^{*}(n-r)\right| \\
\Delta V_{22}(n)= & V_{22}(n+1)-V_{22}(n) \\
\leq & -\left(\frac{1}{\theta_{2}(n)}-\left|\frac{1}{\theta_{2}(n)}-q(n)\right|\right)\left|y(n)-y^{*}(n)\right|+p(n)\left|x(n)-x^{*}(n)\right| \\
& \sum_{r=0}(r)\left|x(n-r)-x^{*}(n-r)\right|+\sum_{r=0}^{M} k_{4}(r)\left|y(n-r)-y^{*}(n-r)\right| \\
&
\end{aligned}
$$


where $\theta_{2}(n)$ is between $y(n)$ and $y^{*}(n)$. Consequently, one has

$$
\begin{aligned}
\Delta V_{2}(n)= & \Delta V_{21}(n)+\Delta V_{22}(n) \\
\leq & -\left(\frac{1}{\theta_{2}(n)}-\left|\frac{1}{\theta_{2}(n)}-q(n)\right|-\sum_{r=0}^{M} k_{4}(r)\right)\left|y(n)-y^{*}(n)\right| \\
& +\left(p(n)+\sum_{r=0}^{M} k_{3}(r)\right)\left|x(n)-x^{*}(n)\right| .
\end{aligned}
$$

Now, we consider a Lyapunov-like discrete function $V(n)$ defined by

$$
V(n)=V_{1}(n)+V_{2}(n)
$$

According to (2.8) and (2.11), there exists an $n^{*} \in \mathbb{Z}^{+}$such that $x(n) \leq M_{1}+\varepsilon, y(n) \leq M_{2}+\varepsilon$ for $n \geq n^{*}$ and any positive constant $\varepsilon$. Obviously, $V(n) \geq 0$ for all $n \in \mathbb{Z}^{+}$and $V\left(n^{*}+M\right)<+\infty$. In view of (3.1), we choose a sufficient small $\varepsilon>0$ such that

$$
\begin{aligned}
& \min \left\{b^{l}, \frac{2}{M_{1}+\varepsilon}-b^{u}\right\}-(M+1)\left(k_{1}^{u}+k_{3}^{u}\right)-p^{u} \geq \eta, \\
& \min \left\{q^{l}, \frac{2}{M_{2}+\varepsilon}-q^{u}\right\}-(M+1)\left(k_{2}^{u}+k_{4}^{u}\right)-c^{u} \geq \eta
\end{aligned}
$$

Therefore, combining (3.9) with (3.13) for all $n \geq n^{*}+M$, we obtain

$$
\begin{aligned}
\Delta V(n)= & \Delta V_{1}(n)+\Delta V_{2}(n) \\
\leq & -\left(\frac{1}{\theta_{1}(n)}-\left|\frac{1}{\theta_{1}(n)}-b(n)\right|-\sum_{r=0}^{M} k_{1}(r)-\sum_{r=0}^{M} k_{3}(r)-p(n)\right)\left|x(n)-x^{*}(n)\right| \\
& -\left(\frac{1}{\theta_{2}(n)}-\left|\frac{1}{\theta_{2}(n)}-q(n)\right|-\sum_{r=0}^{M} k_{4}(r)-\sum_{r=0}^{M} k_{2}(r)-c(n)\right)\left|y(n)-y^{*}(n)\right| \\
\leq & -\left(\min \left\{b^{l}, \frac{2}{M_{1}+\varepsilon}-b^{u}\right\}-(M+1)\left(k_{1}^{u}+k_{3}^{u}\right)-p^{u}\right)\left|x(n)-x^{*}(n)\right| \\
& -\left(\min \left\{q^{l}, \frac{2}{M_{2}+\varepsilon}-q^{u}\right\}-(M+1)\left(k_{2}^{u}+k_{4}^{u}\right)-c^{u}\right)\left|y(n)-y^{*}(n)\right| \\
\leq & -\eta\left(\left|x(n)-x^{*}(n)\right|+\left|y(n)-y^{*}(n)\right|\right) .
\end{aligned}
$$

Summing both sides of (3.16) from $n^{*}+M$ to $n$, it derives that

$$
\sum_{s=n^{*}+M}^{n} \Delta V(s)=\sum_{s=n^{*}+M}^{n}(V(s+1)-V(s)) \leq-\eta \sum_{s=n^{*}+M}^{n}\left(\left|x(s)-x^{*}(s)\right|+\left|y(s)-y^{*}(s)\right|\right),
$$




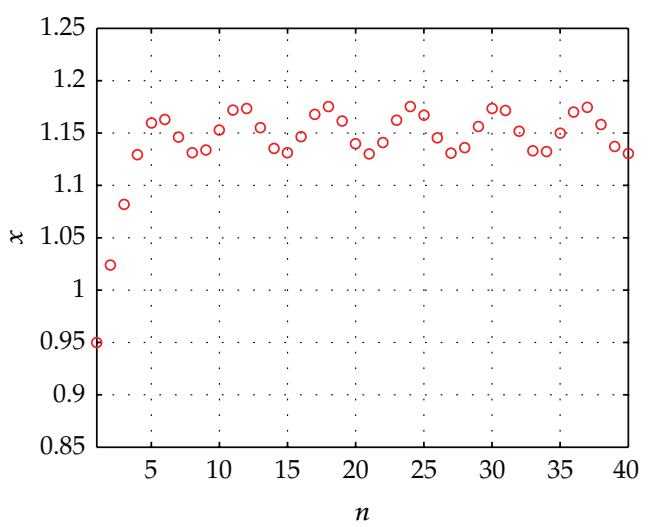

(a)

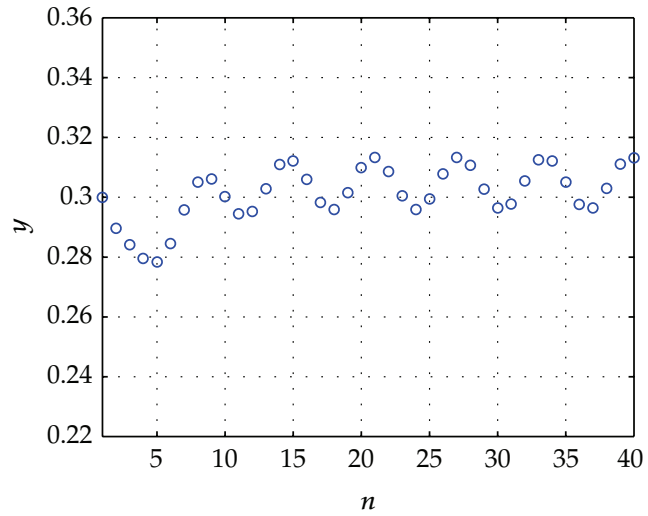

(b)

Figure 1: Permanence of (1.9) with $\left\{(x(-1), y(-1))^{\top}\right\}=\left\{(1.0,0.29)^{\top}\right\}$ and $\left\{(x(0), y(0))^{\top}\right\}=\left\{(0.95,0.3)^{\top}\right\}$. (a) Time series of $x$ for $n \in[0,40]$. (b) Time series of $y$ for $n \in[0,40]$.

which implies that

$$
\begin{aligned}
V(n+1)+\eta & \sum_{s=n^{*}+M}^{n}\left(\left|x(s)-x^{*}(s)\right|+\left|y(s)-y^{*}(s)\right|\right) \leq V\left(n^{*}+M\right), \quad \text { for any } n \geq n^{*}+M, \\
& \sum_{n=n^{*}+M}^{+\infty}\left(\left|x(n)-x^{*}(n)\right|+\left|y(n)-y^{*}(n)\right|\right) \leq \frac{V\left(n^{*}+M\right)}{\eta}<+\infty,
\end{aligned}
$$

from which we conclude that

$$
\lim _{n \rightarrow+\infty}\left(\left|x(n)-x^{*}(n)\right|+\left|y(n)-y^{*}(n)\right|\right)=0
$$

that is,

$$
\lim _{n \rightarrow+\infty}\left|x(n)-x^{*}(n)\right|=0, \quad \lim _{n \rightarrow+\infty}\left|y(n)-y^{*}(n)\right|=0 .
$$

According to Definition 1.2, this result implies that $\left\{(x(n), y(n))^{\top}\right\}$ is global attractive. The proof is complete.

\section{An Example and Its Numerical Simulations}

In this paper, we have investigated the asymptotic behaviors of a delayed nonautonomous predator-prey difference system. Sufficient conditions which guarantee the permanence of system and global attractivity of positive solutions of system are obtained, respectively. The theoretical results are substantiated by the following example and numerical results. 


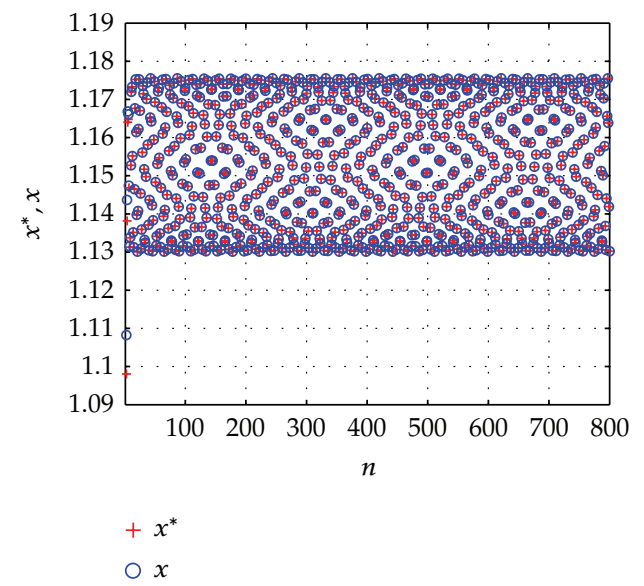

(a1)

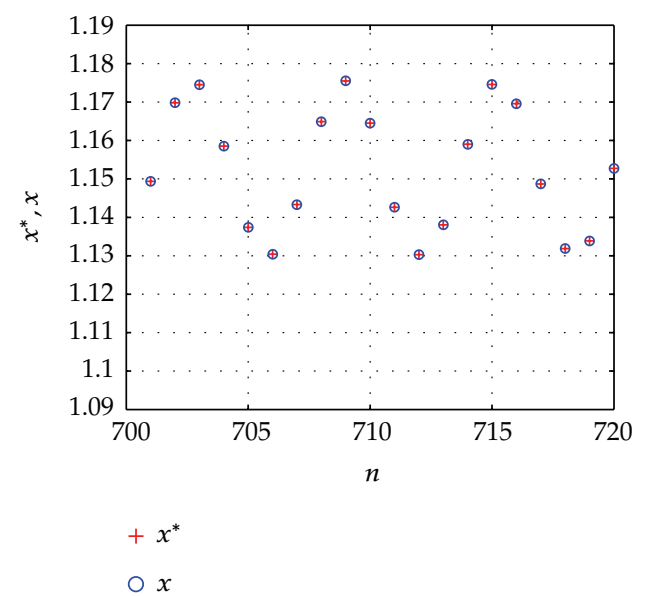

(a2)

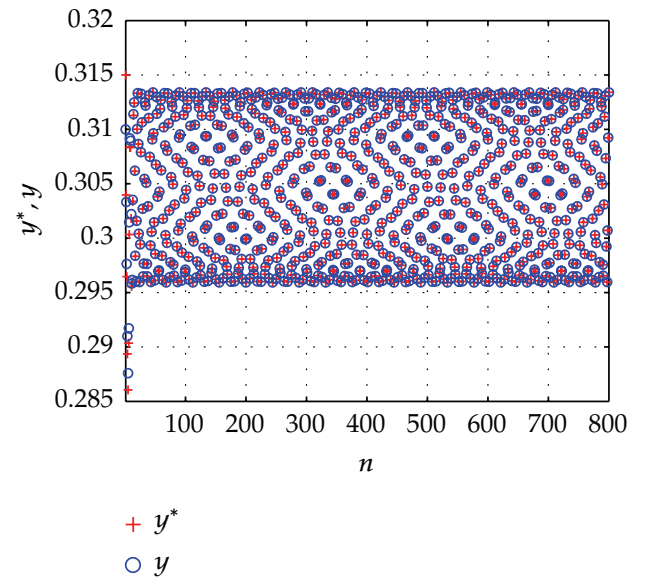

(b1)

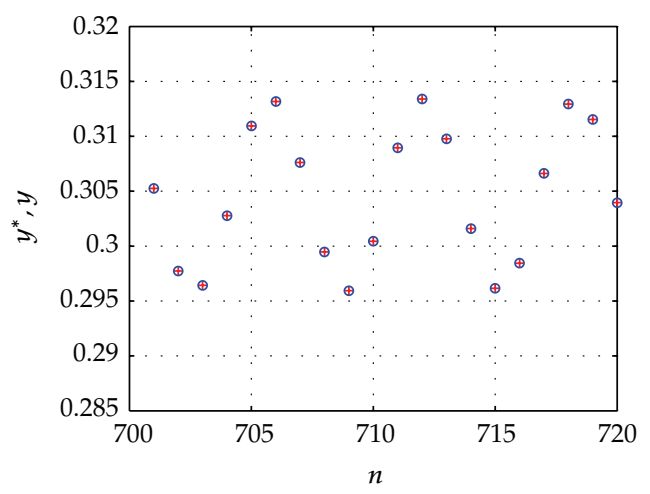

$+y^{*}$

○ $y$

(b2)

Figure 2: Dynamic behaviors of (1.9) with different initial values. (a1) and (a2) show $x^{*}, x$ with $x^{*}(-1)=$ 1.06, $x^{*}(0)=1.0, x(-1)=1.04, x(0)=1.03$ for $k \in[0,800]$ and $k \in[700,720]$, respectively. (b1) and (b2) show $y^{*}, y$ with $y^{*}(-1)=0.32, y^{*}(0)=0.315, y(-1)=0.315, y(0)=0.31$ for $k \in[0,800]$ and $k \in[700,720]$, respectively.

Example 4.1. Consider the following system:

$$
\begin{gathered}
x(n+1)=x(n) \exp \{0.5+0.01 \sin n-(0.38+0.02 \sin n) x(n)-(0.02+0.01 \sin n) y(n) \\
-0.024[x(n)+x(n-1)]-0.001[y(n)+y(n-1)]\}, \\
y(n+1)=y(n) \exp \{-0.015-0.005 \sin n+(0.26+0.03 \sin n) x(n)-(0.97+0.02 \sin n) y(n) \\
+0.01[x(n)+x(n-1)]-0.02[y(n)+y(n-1)]\} .
\end{gathered}
$$

Obviously,

$$
\Delta_{1} \approx 0.5175>0, \quad \Delta_{2} \approx 0.3875>0, \quad \Delta_{3} \approx 0.1347>0 .
$$


It is easy to verify that the assumptions of Theorem 2.3 are satisfied, so system (4.1) is permanent (see Figure 1). Furthermore, a calculation shows that

$$
\begin{aligned}
& \min \left\{b^{l}, \frac{2}{M_{1}}-b^{u}\right\}-(M+1)\left(k_{1}^{u}+k_{3}^{u}\right)-p^{u} \approx 0.0020>0.001 \\
& \min \left\{q^{l}, \frac{2}{M_{2}}-q^{u}\right\}-(M+1)\left(k_{2}^{u}+k_{4}^{u}\right)-c^{u} \approx 0.8780>0.001
\end{aligned}
$$

According to Theorem 3.1, any positive solution $\left\{(x(n), y(n))^{\top}\right\}$ of system (4.1) is global attractive (see Figure 2). From Figures 2(a1) and 2(a2), we see that $x$ with $x(0)=1.03$ tends to $x^{*}$ with $x^{*}(0)=1.0$. Similarly, from Figures 2(b1) and 2(b2), we see that $y$ with $y(0)=0.31$ tends to $y^{*}$ with $y^{*}(0)=0.315$.

\section{Acknowledgments}

This work is supported by the Key Project of Chinese Ministry of Education (no. 210134), the Innovation Term of Educational Department of Hubei Province of China (no. T200804), and the Project of Key Laboratory of Biological Resources Protection and Utilization of Hubei Province. The authors would like to thank the anonymous referees for their helpful comments which improved the presentation of this work.

\section{References}

[1] J. M. Cushing, "Periodic solutions of two species interaction models with lags," Mathematical Biosciences, vol. 31, no. 1-2, pp. 143-156, 1976.

[2] J. K. Hale, Functional Differential Equations, Springer, New York, NY, USA, 1971.

[3] J. J. Levin and J. A. Nohel, "On a nonlinear delay equation," Journal of Mathematical Analysis and Applications, vol. 8, pp. 31-44, 1964.

[4] A. Leung, "Conditions for global stability concerning a prey-predator model with delay effects," SIAM Journal on Applied Mathematics, vol. 36, no. 2, pp. 281-286, 1979.

[5] S. Q. Liu, E. Beretta, and D. Breda, "Predator-prey model of Beddington-DeAngelis type with maturation and gestation delays," Nonlinear Analysis. Real World Applications, vol. 11, no. 5, pp. 4072-4091, 2010.

[6] W. Zuo and J. Wei, "Stability and Hopf bifurcation in a diffusive predatory-prey system with delay effect," Nonlinear Analysis. Real World Applications, vol. 12, no. 4, pp. 1998-2011, 2011.

[7] Z. J. Liu, R. H. Tan, and L. S. Chen, "Global stability in a periodic delayed predator-prey system," Applied Mathematics and Computation, vol. 186, no. 1, pp. 389-403, 2007.

[8] C.-H. Zhang, X.-P. Yan, and G.-H. Cui, "Hopf bifurcations in a predator-prey system with a discrete delay and a distributed delay," Nonlinear Analysis. Real World Applications, vol. 11, no. 5, pp. 4141-4153, 2010.

[9] Z. Luo, B. Dai, and Q. Zhang, "Existence of positive periodic solutions for an impulsive semi-ratiodependent predator-prey model with dispersion and time delays," Applied Mathematics and Computation, vol. 215, no. 9, pp. 3390-3398, 2010.

[10] Z.-P. Ma, H.-F. Huo, and C.-Y. Liu, “Stability and Hopf bifurcation analysis on a predator-prey model with discrete and distributed delays," Nonlinear Analysis. Real World Applications, vol. 10, no. 2, pp. 1160-1172, 2009.

[11] L. Chen and F. Chen, "Dynamic behaviors of the periodic predator-prey system with distributed time delays and impulsive effect," Nonlinear Analysis. Real World Applications, vol. 12, no. 4, pp. 2467-2473, 2011.

[12] W. Yan and J. R. Yan, "Periodicity and asymptotic stability of a predator-prey system with infinite delays," Computers \& Mathematics with Applications, vol. 60, no. 5, pp. 1465-1472, 2010. 
[13] X. X. Liu, "A note on the existence of periodic solutions in discrete predator-prey models," Applied Mathematical Modelling, vol. 34, no. 9, pp. 2477-2483, 2010.

[14] R. P. Agarwal, Difference Equations and Inequalities: Theory, Methods, and Application, Marcel Dekker, New York, NY, USA, 2000.

[15] H. I. Freedman, Deterministic Mathematical Models in Population Ecology, Marcel Dekker, New York, NY, USA, 1980.

[16] W. Qin and Z. Liu, "Asymptotic behaviors of a delay difference system of plankton allelopathy," Journal of Mathematical Chemistry, vol. 48, no. 3, pp. 653-675, 2010.

[17] W. J. Qin, Z. J. Liu, and Y. P. Chen, "Permanence and global stability of positive solutions of a discrete competitive system," Discrete Dynamics in Nature and Society, vol. 2009, Article ID 830537, 13 pages, 2009.

[18] J. D. Murray, Mathematical Biology, vol. 19, Springer, Berlin, Germany, 1989.

[19] X. T. Yang, “Uniform persistence and periodic solutions for a discrete predator-prey system with delays," Journal of Mathematical Analysis and Applications, vol. 316, no. 1, pp. 161-177, 2006. 


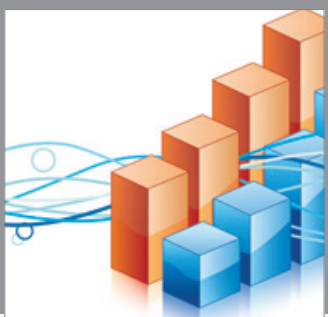

Advances in

Operations Research

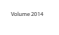

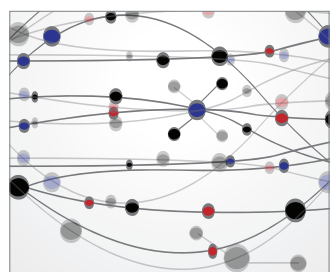

\section{The Scientific} World Journal
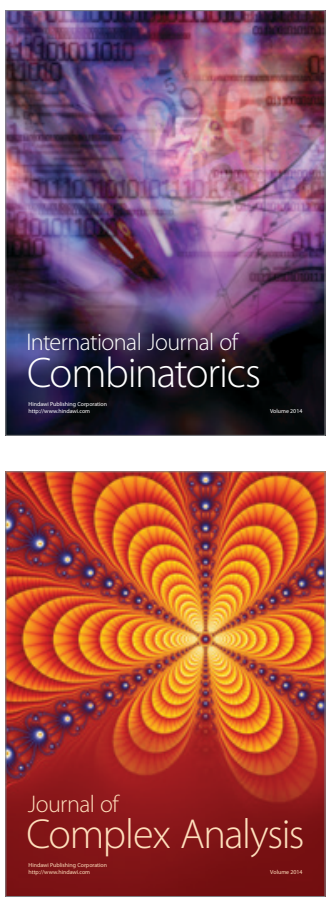

International Journal of

Mathematics and

Mathematical

Sciences
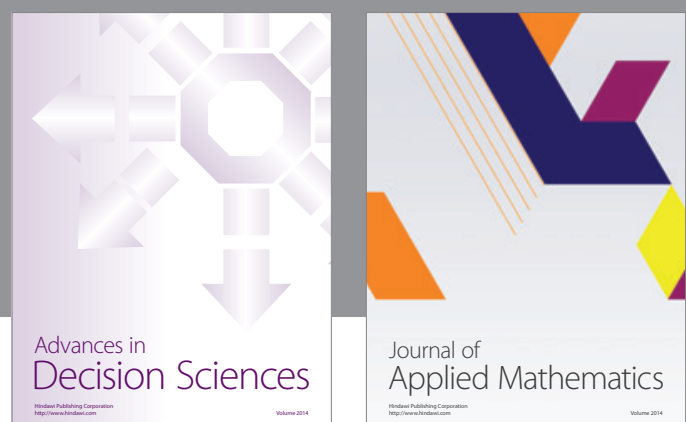

Journal of

Applied Mathematics
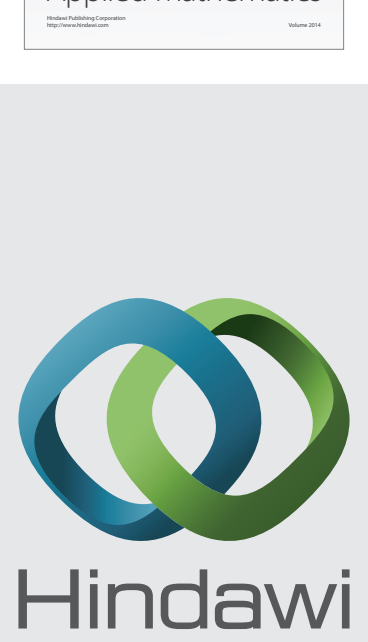

Submit your manuscripts at http://www.hindawi.com
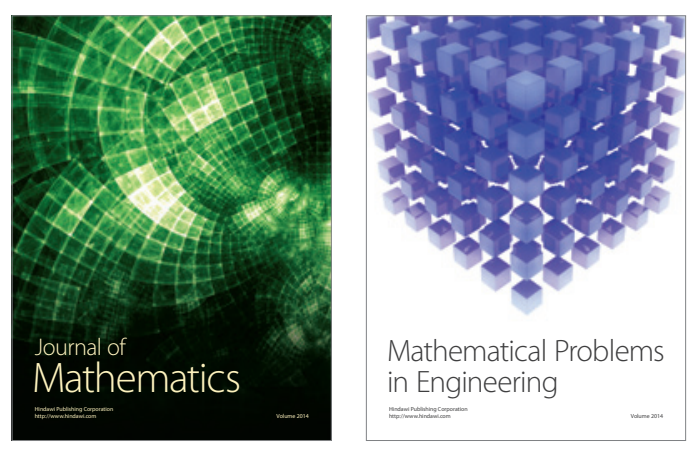

Mathematical Problems in Engineering
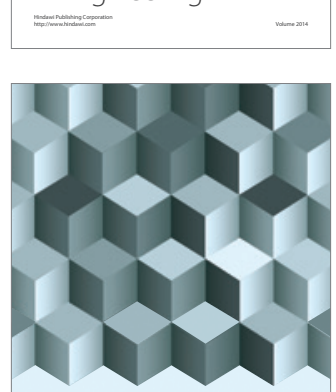

Journal of

Function Spaces
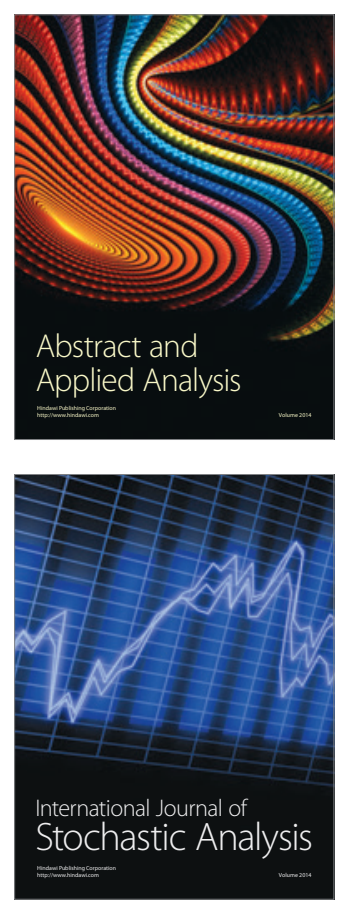

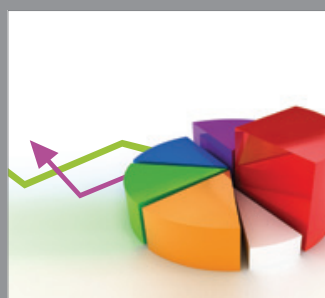

ournal of

Probability and Statistics

Promensencen
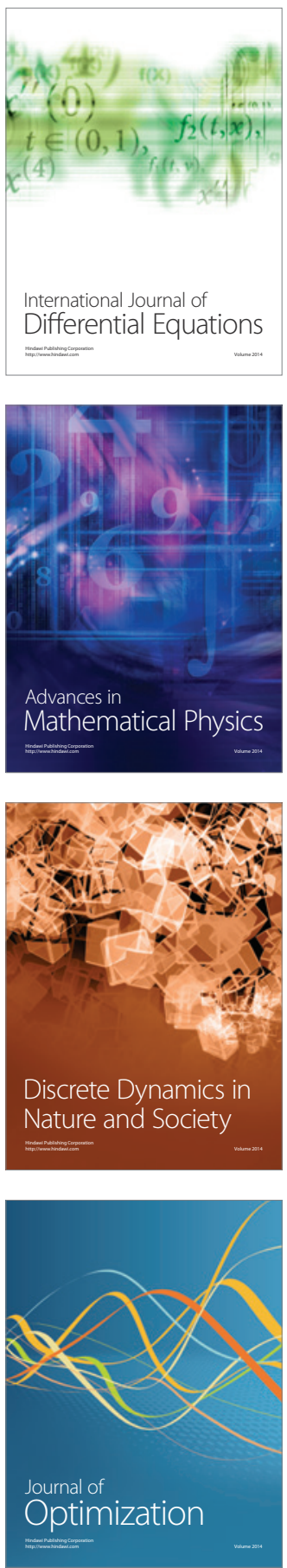